\section{(D) Check for updates}

Cite this: Org. Chem. Front., 2020, 7, 2776

Received 20th May 2020,

Accepted 4th August 2020

DOI: $10.1039 / \mathrm{dOqo00613k}$

rsc.li/frontiers-organic

\title{
Organic micro/nanoscale materials for photonic barcodes
}

\author{
Yue Hou, ${ }^{a}$ Zhenhua Gao, ${ }^{b}$ Yong Sheng Zhao (iD ${ }^{a}$ and Yongli Yan (D)*a
}

Photonic barcodes have attracted extensive attention due to their promising applications in multiplexed high throughput bioassays, item tracking, information security, etc. Organic luminescent materials, capable of assembling into various micro/nanostructures as a result of their outstanding processability, afford a fundamental model for designing novel micro/nanoscale photonic barcodes. In this highlight, we review recent advances in micro/nanoscale photonic barcodes based on organic materials. This highlight starts by introducing the significance of tiny photonic barcodes and discussing why organics have become dominant. Then we present the research progress on organic photonic barcodes in terms of a series of strategies including graphical encoding, spectroscopic encoding and a combination of them both. In the following part, we focus on the construction of covert photonic barcodes based on nearinfrared luminescence and responsive excited state processes, which have shown great potential in advanced anti-counterfeiting. Finally, we present our views on the current challenges and the future development of organic micro/nanoscale photonic barcodes. We believe that a comprehensive understanding on this topic would significantly contribute to the advancement of organic photonic barcodes for a broad range of applications.

\section{Introduction}

Photonic barcodes have been demonstrated to have a great value for tracking and identifying a vast amount of information of the macroscopic world in our daily lives. Traditional barcodes contain a series of black bars with different widths representing consecutive digits, and thus countless codes can be generated by systematically changing the number of bars, width, and spaces between adjacent bars, showing huge coding capacity. These macroscopic barcodes have been widely applied in different products including stationery products and letters, as well as in healthcare settings, and they permit a rapid, simple, and accurate identification of the target. ${ }^{1}$ However, with the significant development of the manufacturing industry and life sciences, there are increasing demands in labelling and tracking micro/nanoscale targets ranging from cells and biomolecules to chip components. ${ }^{2}$ Therefore, the macroscale feature of traditional barcodes has become a limiting factor for these applications. Compared with macroscopic barcodes, nano or micro barcodes can greatly improve the storage capacity and accuracy because of their small size. In

\footnotetext{
${ }^{a}$ Key Laboratory of Photochemistry, Institute of Chemistry, Chinese Academy of Sciences, Beijing 100190, China. E-mail: ylyan@iccas.ac.cn

${ }^{b}$ School of Materials Science \& Engineering, Qilu University of Technology (Shandong Academy of Sciences), 3501 Daxue Road, Changqing District, Jinan 250353,

Shandong Province, China
}

this regard, photonic barcodes with a small footprint and unique identifiability have shown great potential for multiplexed high-throughput bioassays, tracking systems and anticounterfeiting applications. ${ }^{3}$ Nowadays, a broad range of optical micro/nanomaterials, including metallic nanoparticles, semiconductor quantum dots, lanthanide-doped nanoparticles, and structural color materials, are being widely explored for their utility in optical encryption and anticounterfeiting. ${ }^{4}$ However, these barcodes often suffer from some inevitable drawbacks ${ }^{5}$ including complex preparation, high-cost processing and low-level readout, which further hinder their practical applications.

Organic luminescent materials, with weak intermolecular interactions and outstanding ability to be flexibly processed into diverse micro/nanostructures, ${ }^{6}$ offer a fundamental model for designing novel micro/nanoscale photonic barcodes. ${ }^{7}$ The emission wavelengths of various dyes can be easily tuned from ultraviolet (UV) to near-infrared (IR) through molecular design. ${ }^{8}$ Besides the photoluminescence (PL) spectra, Raman scattering resulting from the vibrational signatures of molecules affords another type of spectrum with narrow spectral bands and high resistance toward fluorescence backgrounds. ${ }^{9}$ These versatile spectra from organic molecules enable the generation of a large library of encoding elements, providing a good platform to develop spectroscopic encoding (Fig. 1). ${ }^{10}$ Furthermore, the outstanding chemical versatility and flexible processability of organic materials allow for integrating 


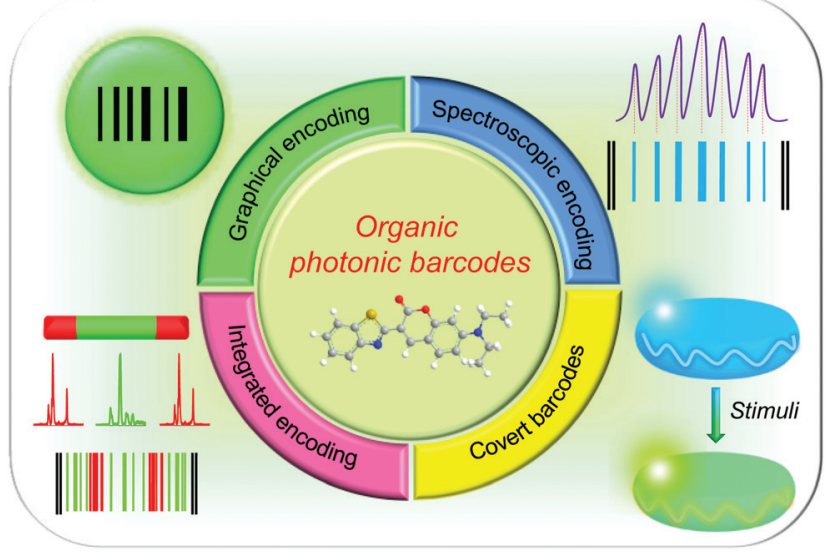

Fig. 1 Graphic illustration of characteristics and function engineering to develop organic micro/nanoscale photonic barcodes.

different identifiable patterns in a single micro/nanostructure, ${ }^{11}$ which presents great potential in designing graphical codes.

Moreover, the coexistence of abundant spectra and excellent compatibility in organic materials enables effective integration of the spectroscopic and graphical encoding in a single microstructure system, where the advantages of identifiable organic microstructures and distinguishable spectral characteristics can simultaneously be utilized, thereby greatly enlarging the encoding capacity and boosting the security. Last but not least, the abundant excited state processes and energy levels in organic materials, including photoisomerization, ${ }^{12}$ exciton conversion/transfer, ${ }^{13}$ energy upconversion, ${ }^{14}$ etc. exhibit smart responsiveness to external stimuli ${ }^{15}$ affording an opportunity to develop covert photonic barcodes, which are of great significance in optical data recording, anti-counterfeiting, and security labels. ${ }^{16}$ Therefore, it is increasingly important to summarize this growing research field, with great emphasis on encoding strategies and the corresponding structure-property relationships of the organic micro/nanostructures to promote future development.

In this highlight, we review the recent advances in the field of organic micro/nanoscale photonic barcodes, with main focus on diverse optical encoding techniques based on identifiable organic microstructures, distinguishable spectral characteristics and controlled excited-state processes toward the construction of covert photonic barcodes. A systematic discussion will be conducted on the evolution of organic photonic barcodes in terms of a series of strategies. It starts with graphical encoding, spectroscopic encoding and the combination of both in organic materials, which can greatly enlarge the encoding capacity. Then, we introduce the construction of the covert photonic barcodes based on the near-infrared (NIR) luminescence and smart responsiveness to external stimuli, which have demonstrated great potential in the application of advanced anti-counterfeiting. Finally, we present our views on the current challenges and future developments of organic micro/nanoscale photonic barcodes. We hope that this highlight will make a contribution to the advancement of organic photonic barcodes for a broad spectrum of applications.

\section{Photonic barcodes with organic micro/nanomaterials}

Organic luminescent materials are endowed with versatile encoding elements due to their abundant spectra and excellent compatibility. This section discusses different encoding mechanisms to produce micro/nanoscale photonic barcodes based on the unique characteristics of organic materials and the benefits/limitations of each type.

\subsection{Graphical encoding}

Graphical encoding is a common coding approach on the basis of optically detectable patterns, and provides a relatively large encoding capacity through modulating the features of the identifiable elements. ${ }^{17}$ The most well-established scheme for graphical encoding was striped metal nanowires, which were prepared by sequentially electrodepositing different metal ions into individual pores of hard templates. ${ }^{18}$ Therefore, the as-fabricated striped metal rods, composed of different metals such as gold $(\mathrm{Au})$, silver $(\mathrm{Ag})$, cobalt $(\mathrm{Co})$, platinum $(\mathrm{Pt})$, nickel $(\mathrm{Ni})$, and copper $(\mathrm{Cu})$, show differential optical reflectivity corresponding to the sequential metal composition, permitting an effective identification of the barcodes. However, the encoding capacity in these composition-related methods is heavily restricted by the complicated preparation methods and relatively limited metals with differential optical reflectivity.

2.1.1 Graphical encoding based on organic multicolor heterojunctions. Compared with the indistinguishable optical reflectivity in metal barcodes, visual photoluminescence is much more intuitive. Organic one-dimensional (1D) segmented structures with domain-controlled colors are superior for achieving graphical encoding. ${ }^{19}$ Benefitting from the excellent flexibility and molecular compatibility, different organic molecules can assemble into hybrid microstructures, making it possible to integrate different identifiable patterns in a single microstructure, which provides a good platform to design excellent multicolor graphical encoding.

Driven by the weak intermolecular interactions, organic molecules tend to self-assemble into various micro/nanostructures. Multicomponent structures can be obtained by carefully controlling the intermolecular interaction and molecular recognition during the cooperative assembly processes. The as-prepared products exhibit distinct emission patterns, which can be viewed as identifiable elements for multicolor barcodes. Zhao and co-workers reported the fabrication of heterostructures with axially tunable compositions. ${ }^{20}$ 2,4,5Triphenylimidazole (TPI) with a planar molecule structure was selected as the host molecule and two other molecules, a sphere-like organometallic complex iridium(III) bis(2-phenyl benzothiozolato-N,C2 $)\left((\mathrm{BT})_{2} \operatorname{Ir}(\mathrm{acac})\right)$ and a planar molecule 9,10-bis (phenylethynyl) anthracene (BPEA), were chosen as 

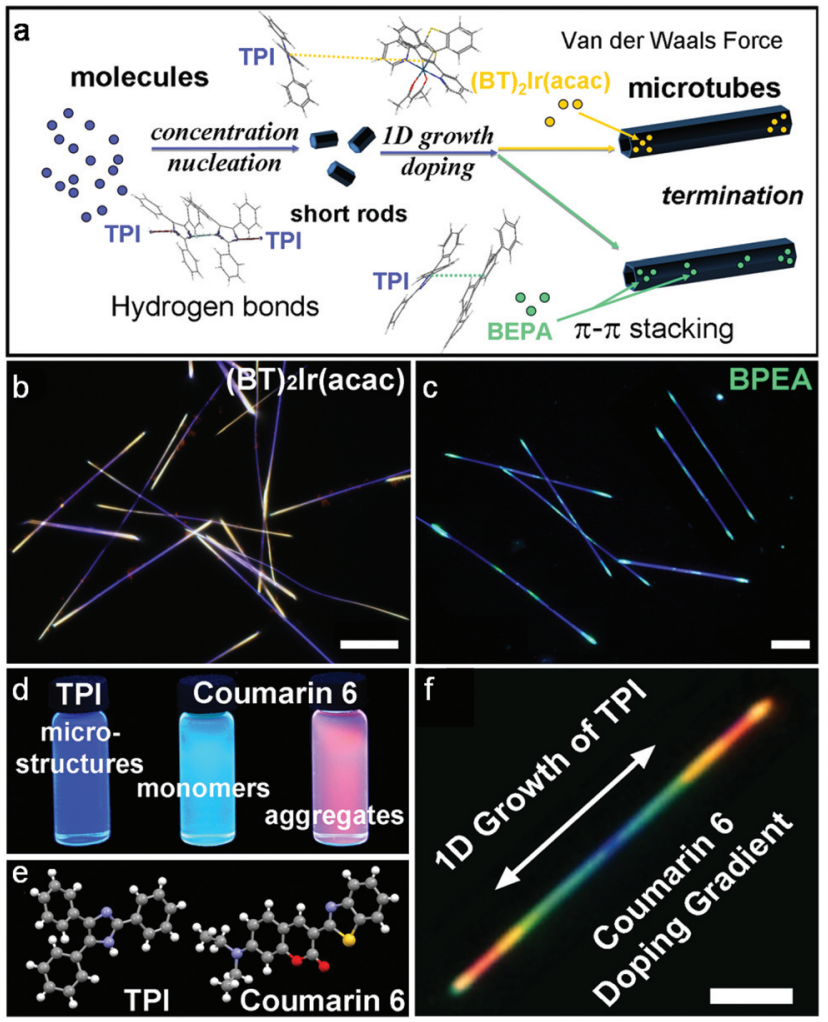

Fig. 2 (a) Schematic illustration of the formation of organic multisegmented microstructures. TPI was used to build 1D tubular host microstructures due to its strong hydrogen bonds along the $c$ crystal axis, while $(\mathrm{BT})_{2} \operatorname{Ir}(\mathrm{acac})$ and BPEA were selected as two typical kinds of guest compounds. (b and c) PL microscopy images of the triblock $(B T)_{2} \operatorname{Ir}(\mathrm{acac})$ and multiblock BPEA doped TPI microtubes. Scale bars are $20 \mu \mathrm{m}$. (d) Digital photographs of suspensions of TPI microstructures (blue), coumarin 6 monomers (green) and aggregates (red) under UV radiation. The red emission from coumarin 6 aggregates originates from the charge transfer (CT) state. (e) Molecular structures of TPI (donor) and coumarin 6 (acceptor). (f) PL microscopy image of coumarin 6 doped TPI microtubes with spatial full-color emission. The scale bar is $5 \mu \mathrm{m}$. $(\mathrm{a}-\mathrm{c})$ Reproduced with permission from ref. 20. Copyright 2012, Wiley-VCH. (d and f) Reproduced with permission from ref. 22. Copyright 2013, Wiley-VCH.

two typical guest molecules, respectively (Fig. 2a). Because of the weak intermolecular interactions (van der Waals force) and the complete mismatch in molecular structures between TPI and sphere-like $(\mathrm{BT})_{2} \operatorname{Ir}(\mathrm{acac})$, the concentrated $(\mathrm{BT})_{2} \operatorname{Ir}(\mathrm{acac})$ guest molecules aggregate together with the epitaxially growing TPI matrix to terminate the $1 \mathrm{D}$ growth of the TPI tubes, finally forming barcode-like microtubes with two doped orange blocks as the ends (Fig. 2b). In comparison, the planar BPEA and TPI molecules with a strong $\pi-\pi$ stacking interaction allow for the continuous growth of the TPI molecules to form two other extended blue blocks even if the BPEA ends its aggregation. With the continuous evaporation of the solvent, BPEA aggregates again along the $1 \mathrm{D}$ growth direction (Fig. 2c). The alternate growth of TPI and BPEA results in the multiblock structures with tunable colors, thus greatly enlarging the encoding capacity of graphical encoding.
The versatile color design of organic materials enables the integration of a large library of optical encoding elements into a single microstructure, providing great opportunities to realize multicolor photonic barcodes with full color blocks. It is demonstrated that the emissive color of organic materials not only depends on their molecule structures, but is also affected by its aggregate environment. ${ }^{21}$ Here, we take 3-(2-benzothiazolyl)-7-diethylaminocoumarin (coumarin 6) as an example. Besides the intrinsic green fluorescence from the coumarin 6 monomer, red emission can be observed from its aggregates as a result of the charge transfer (CT) state (Fig. 2d). Benefiting from the superior compatibility of TPI crystalline matrices to coumarin 6 molecules, when coumarin 6 was adopted as the guest compound, barcode blocks with full color range were obtained (Fig. 2e). ${ }^{22}$ The gradually varying dispersion of coumarin 6 clusters induces the full-colored visible luminescence along these microtubes due to the energy transfer from TPI to coumarin 6 and the aggregation-induced spectral shift of coumarin 6 (Fig. 2f). Therefore, the RGB colors in a single luminescent micro/nanomaterial could be accomplished by the controllable doping of coumarin 6 into TPI structures, manifesting the infinite capacity of such barcodes.

However, owing to the gradient axial doping of guest molecules, the interfaces between adjacent blocks in multicolor heterostructures are not clear, which may further affect the precise acquisition of the barcode information. Therefore, constructing multisegmented heterostructures with a clear interface would be beneficial for the accurate identification of the barcodes. Metallophosphors with similar molecular structures (Fig. 3a) were chosen to prepare multicolor heterostructures with a very sharp color change through collaborative assembly. ${ }^{23}$ The superior structural compatibility between the two components permits the dispersion of the $\mathrm{Ru}$ acceptor into the Ir donor crystalline matrix, which results in materials with high crystallization and tunable emission properties. Multiblock binary heterojunctions of the Ir donor and the Ru acceptor were successfully constructed by carefully controlling the contents of the stock solution using stepwise growth conditions (Fig. 3b and c). These 1D penta-block heterojunctions exhibit multicolor emissions with a very sharp color change. It is worth mentioning that the emission patterns are entirely different when these heterostructured nanorods are irradiated with blue or red light (Fig. 3d and e), implying the possibility of functioning as covert barcodes.

Multiblock heterostructures with changeable color and length generated through carefully controlling the intermolecular interaction would dramatically enlarge the encoding capacity. However, the lack of a distinct border between adjacent segments may lead to complications in the precise identification of the coded information. Metallophosphors with similar molecular structures, capable of assembling into regulated multiblocks with clear boundaries, not only show superiority in accurate information recognition and extraction, but also hold great potential to design covert barcodes.

2.1.2 Graphical encoding based on photobleaching. Organic luminescent materials possessing versatile emission 
a

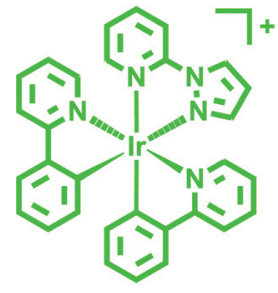

Polypyridyl Ir(III)
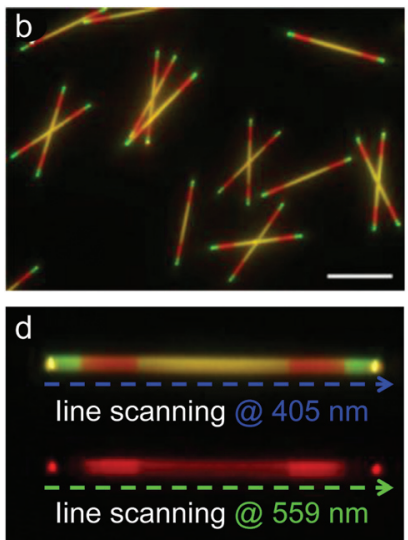

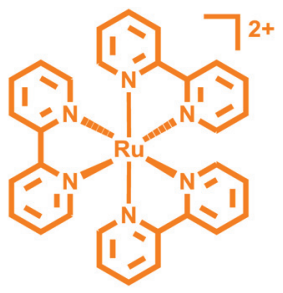

Polypyridyl Ru(III)
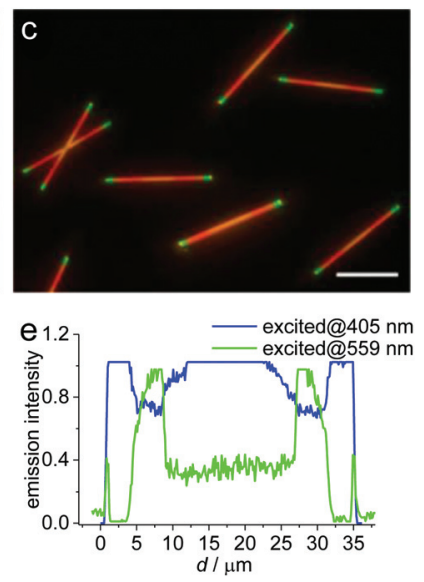

Fig. 3 (a) Chemical structures of the Ir donor and Ru acceptor. The similar molecular structures ensure the collaborative assembly of the two compounds. (b and c) Fluorescence microscopy images of heterojunction nanorods doped with (b) $1 \%$, and (c) $2 \%$ Ru acceptor under UV irradiation, showing multiple-color emissions. Scale bars are $20 \mu \mathrm{m}$. (d) Line scanning along a single nanorod using a confocal laser scanning microscope equipped with 405 and $559 \mathrm{~nm}$ lasers. (e) The corresponding plots of the microarea emission intensity as a function of the distance $d$ along the rod axis. Reproduced with permission from ref. 23. Copyright 2018, American Chemical Society.

colors are superior in graphic encoding for easy visualization. ${ }^{24}$ Inspired by the fact that PL intensities in organic molecules could be freely modulated through light stimuli owing to their flexible molecular skeleton, De Smedt and co-workers developed a novel approach to encode polymer microspheres based on "spatial selective photobleaching", where identifiable patterns can be directly written on the surface of fluorescent microspheres (Fig. 4a and b). ${ }^{25}$ Photobleaching is a photoinduced process where luminescent molecules lose their fluorescence, resulting in a fading of the fluorescent color. Therefore, the emissive and non-emissive areas constitute different graphical patterns with high contrast. As shown in Fig. 4a, barcodes can be clearly written by "spatially selective photobleaching" of the fluorescence in homogeneously fluorescently dyed microspheres. Certain regions at a certain depth in the microspheres were bleached, and the as-obtained barcodes are illustrated in Fig. 4b. Notably, the emission intensity can be utilized as a code element. In this cast, the only difference among all the bars is different bleaching levels, showing the feasibility of intensity encoding. Theoretically, the photobleaching method is able to generate an unlimited number of

a

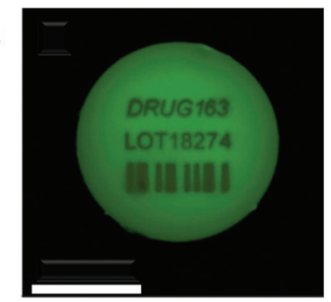

c

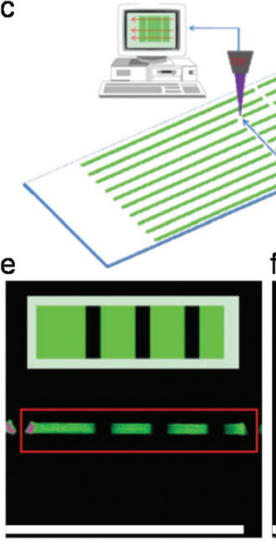

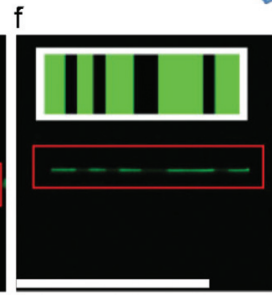

b

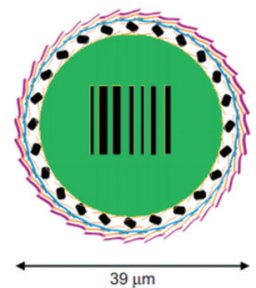

d

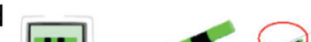

Fig. 4 (a) A typical barcode written via selective photobleaching at the central plane of a homogeneously fluorescent microsphere, where certain regions at a certain depth were bleached. By exposing the regions to be bleached to a high intensity laser beam, the fluorescent dyes lost their fluorescence properties, which gives rise to the code. The scale bar is $20 \mu \mathrm{m}$. (b) Schematic presentation of "bar-encoded" memobeads. (c) Cutting of polymer fibers prepared through electrospinning into fiber pieces by cold ablation. (d) Encoding of the fiber pieces by photobleaching through the use of a scanning laser beam. $(e-g)$ Different barcodes written in the fiber pieces. Note that information can be stored, not only in the width of the bars, but as well in the length of the memofibers. The insets show the barcodes which were written in the fiber pieces, and the red rectangles indicate individual memofibers. Scale bars are $100 \mu \mathrm{m}$. ( $\mathrm{a}$ and b) Reproduced with permission from ref. 25b. Copyright 2007, Wiley-VCH. (c-g) Reproduced with permission from ref. 26. Copyright 2010, Wiley-VCH.

unique codes through changing the width and intensity in barcode information.

However, this method is restricted by the limited central plane of a microsphere for writing a valuable code, because bleaching near the edges becomes less efficient due to the spherical shape. To solve this problem, they further developed highly aligned polymer microfibers instead. ${ }^{26}$ Precisely aligned microfibers through electrospinning are digitally encoded by a laser beam scanning along the longitudinal axis of the fiber pieces (Fig. 4c and d), thus permitting a high coding efficiency and encoding capacity through changing the width and numbers of the bars (Fig. 4e-g). Such encoded micropatterns can find broad applications in high-throughput screening, combinatorial chemistry, and commodity labels to prevent counterfeiting.

Though photobleaching has shown great potential in the encoding area, this method still faces some challenges. For example, fluorescent molecules used here must be fixed in a host material. Otherwise, the bleached code will fade away over 
time resulting from the mutual diffusion between fluorescent and bleached molecules. In addition, the number of encoding features is limited by the particle size, which would restrain the graphical encoding capacity.

As a well-established coding scheme, graphical encoding, which provides a relatively large encoding capacity, has been widely used in both industrial production and our daily life. However, these barcodes have to be placed in a specific orientation in the reading processes owing to the anisotropy of the patterning, which would lead to the increase of operational complexity. ${ }^{27}$

\subsection{Spectroscopic encoding}

Among various encoding strategies explored, spectroscopic encoding is another well-established coding technology, although it is not as intuitive as graphical encoding. Spectroscopic encoding commonly relies on the color, intensity and lifetime information of light emitted from more than one coloring materials such as fluorescent dyes or quantum dots. ${ }^{28}$ Compared with the graphical encoding, spectroscopic encoding is much easier to be recognized without orientation dependence because of the isotropic character of photoluminescence.

2.2.1 Spectroscopic encoding based on multicolor-emitting materials. The versatile spectra from luminescent organic molecules enable the generation of a large library of spectroscopic encoding. However, considering that spectrum-based barcodes are strongly dependent on the excitation efficiencies of different light, multiple light sources of different wavelengths are usually needed during the identification process to maintain a high reading accuracy. ${ }^{29}$ To overcome this obstacle, Xiao et al. proposed an approach to encode microspheres consisting of intramolecular through-bond energy transfer (TBET) molecules (Fig. 5a). ${ }^{30}$ In a TBET cassette, the electron-donor and electron-acceptor groups are connected by a rigid and conjugated linker in the molecular skeleton, which ensures an effective energy-transfer process "through bond". Three boron dipyrromethene (BODIPY) derivatives with well-separated emission bands were chosen to construct the cassettes, which permit highly efficient TBET under single-excitation wavelength. Upon excitation of three fluorophores (BDP, BDP-A1, and BDP-A2) at $480 \mathrm{~nm}$, sharp fluorescence with distinguishable colors was observed. The intensity of each emission maximum can be effectively modulated by simply adjusting the amount of the corresponding fluorophore. Therefore, fluorescence from these microspheres with the TBET feature exhibits tailored emission fingerprints.

Then they set three channels in collecting the emissions from BDP, BDP-A1, and BDP-A2 in microspheres through confocal microscopy images. As shown in Fig. 5b, six kinds of microspheres that show distinct emission intensities in all three channels can be obtained by changing the corresponding loading amounts of each dye. For example, microsphere 1\# solely labeled with BDP shows bright fluorescence in the green channel without emissions in the other two channels. Comparatively, microspheres $5 \#$ doped with all three

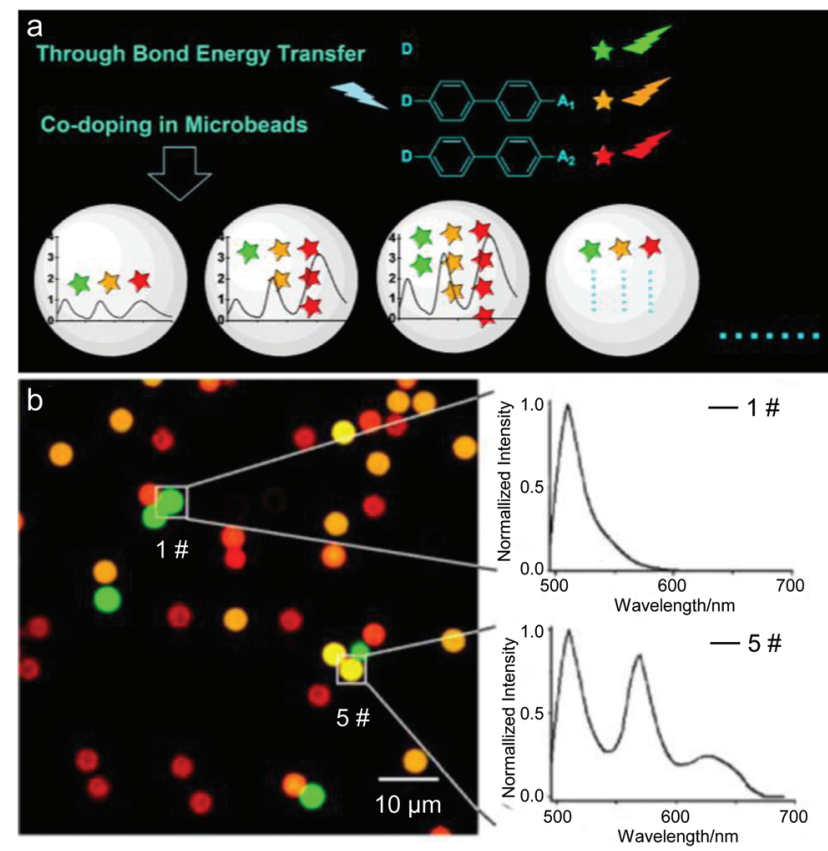

Fig. 5 (a) Schematic illustration of through-bond energy transfer (TBET) as a versatile strategy to develop encoded microspheres. By the connections of two different long wavelength BODIPY dyes to a short wavelength BODIPY dye, two TBET cassettes are constructed. Together with the donor they are loaded into self-produced polystyrene microspheres which demonstrate tailored emission fingerprints upon single excitation of the donor. (b) Overlapped confocal microscopy image of dye-doped fluorescence microspheres in the green channel $\left(\lambda_{\mathrm{em}}=495-555 \mathrm{~nm}\right)$, orange channel $\left(\lambda_{\mathrm{em}}=560-620 \mathrm{~nm}\right)$ and red channel $\left(\lambda_{\mathrm{em}}=\right.$ 655-755 nm). The corresponding spectra of triply doped microspheres (1\# and 5\#) are presented in the right. Reproduced with permission from ref. 30. Copyright 2014, American Chemical Society.

TBET fluorophores exhibits signals in all the three channels. What is more, there is no crosstalk among channels, indicating the coding possibility. The TBET-based encoded microspheres are of practical applicability in multiplex and high throughput assays, which demonstrates satisfactory performances of the novel multicolor encoded microspheres.

2.2.2 Spectroscopic encoding based on microcavity modulation. The broad PL band of different coloring materials is prone to be overlapped; thus the possible number of codes in multicolor-emitting spectroscopic encoding is practically limited by the availability of indicating materials with minimal spectral overlap. ${ }^{31}$ Zhao and co-workers proposed a strategy to design photonic barcodes based on whispering-gallery-mode (WGM) modulations in dye-doped microdisk resonant cavities. $^{32}$ Because photons can be effectively confined in WGM microcavities through continuous total internal reflection along the smooth and curved surfaces, ${ }^{33}$ a microcavity embodying gain materials could emit PL with a series of sharp peaks from WGM cavity modulation when excited locally with a focused laser beam. These recognizable peaks heavily depend on the size, refractive index, and quality factor of the resonator, ${ }^{34}$ thus constituting the fingerprint of a specific reso- 
a
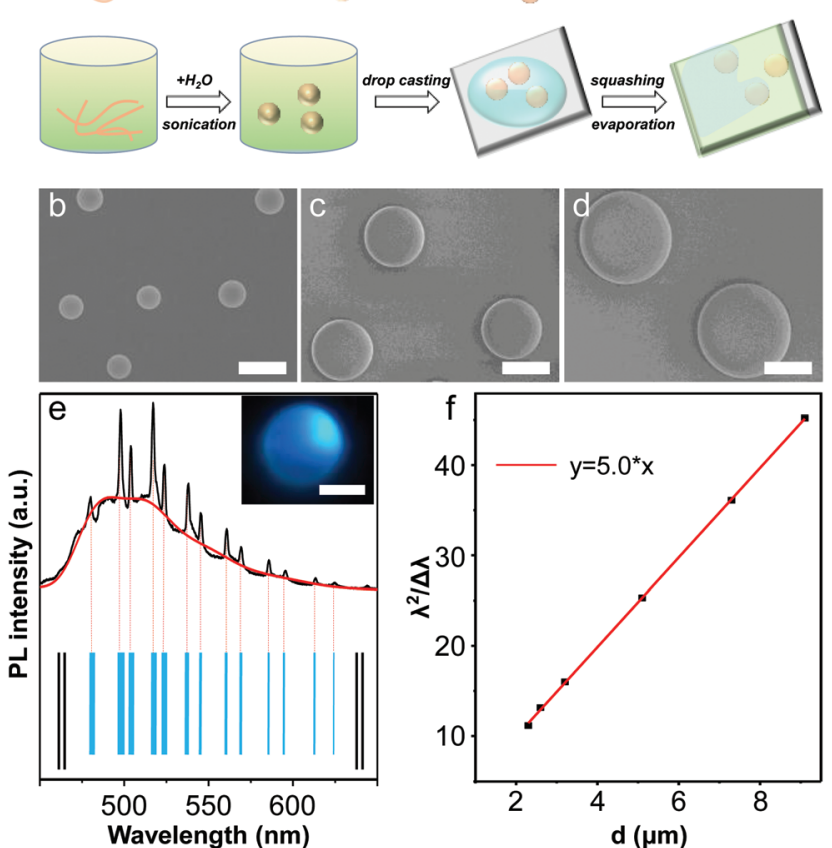

Fig. 6 (a) Schematic diagram of the microdisk fabrication process. The disk-like structures were obtained through controllable evaporation of the solvent of the emulsion solution containing isotropic spherical micelles. During the drying of the PS micelles, a tiny gap consisting of two glass substrates was introduced as a template. (b-d) SEM images of the microdisks prepared with various proportions of water to PS solution, showing flexible preparation of microdisks with varied size. Scale bars are $10 \mu \mathrm{m}$. (e) PL spectra and corresponding photonic barcode from a typical microdisk. The black and red lines represent emissions under excitation of a $405 \mathrm{~nm} \mathrm{CW}$ laser and a UV band of a mercury lamp, respectively. Inset: PL image of the disk. Scale bar is $2 \mu \mathrm{m}$. (f) Relationship between $\lambda 2 / \Delta \lambda$ and the diameter $(d)$ of microdisks, manifesting the formation of WGM resonance. The red line is a linear fit to the function $\lambda 2 / \Delta \lambda=n \pi d$. Reproduced with permission from ref. 32 . Copyright 2017, Wiley-VCH.

nator and providing a larger coding capacity than traditional spectroscopic encoding.

Organic materials possess excellent flexibility and processability, making them an ideal candidate for producing micro-/ nanoscale WGM cavities. ${ }^{35}$ As shown in Fig. 6a, disk-like structures were fabricated through liquid phase assembly. Polystyrene (PS) molecules dissolved in $N, N$-dimethylformamide (DMF) aggregated into independent spherical micelles when a small amount of water was added. During the drying of PS micelles, a tiny gap consisting of two glass substrates was introduced as a template, and spherical micelles got squashed. The microdisk diameter could be finely tuned through changing the proportion of added water to PS solution (Fig. 6b-d). Under a focused laser beam, a bright ring shape pattern was observed at the boundary of the microdisk, which indicated that the microdisk formed a typical WGM feedback resonant cavity. Moreover, a series of sharp peaks were found to superimpose upon the broad emission spectrum collected from the edge of the microdisk. The WGM-modulated recog- nizable spectrum affords a good platform to encode and identify each microdisk. In general, a typical barcode contains a series of black bars with different widths representing consecutive digits. Here, barcodes are defined as follows: each solid bar is located in the wavelength position of the corresponding mode in the PL spectrum, and the bar width is defined by the relative fluorescence intensity of the same wavelength (Fig. 6e). According to the coding rule, a specific barcode can be deduced from the modulated PL spectrum.

In the modulated spectrum, the mode spacing $\Delta \lambda$ and the diameter $d$ of the corresponding microcavity satisfy the equation $\lambda 2 / \Delta \lambda=n \pi d$, where $\lambda$ is the wavelength of the confined light and $n$ is the group refractive index. ${ }^{36}$ The relationship between $\lambda 2 / \Delta \lambda$ and $d$ shown in Fig. $6 f$ indicates that countless barcodes can be produced through controllable varying of diameters of the microdisks. The coding capacity can also be further enlarged via selection of other organic dyes because of their diverse PL emission.

2.2.3 Spectroscopic encoding based on Raman scattering. For organic fluorescent dyes, photobleaching ${ }^{37}$ is an unavoidable difficulty since their debut, and is fatal to the reliability of photonic barcodes. In contrast, Raman scattering, ${ }^{38}$ involving vibrational energy being gained by a molecule as incident photons from a laser are shifted to lower energy, could provide a more stable spectrum with a number of relatively narrow peaks. Each peak corresponds to a specific molecular bond vibration, enabling accurate readout of the as-generated photonic barcodes based on Raman scattering.

Wang and co-workers developed a wavenumber-intensity joint spectral encoding method on the basis of surface enhanced Raman scattering (SERS). ${ }^{39}$ Three kinds of Raman reporters, 1,4-benzenedithiol (BDT), 2-naphthalenethiol (2-NAT) and 4-methoxythiophenol (4-MT), were used, and their Raman peaks at 730,767 , and $794 \mathrm{~cm}^{-1}$ were used as the encoding bands. According to the information on both the wavenumber and intensity of the SERS signals, a total of 19 codes were achieved experimentally with distinguishable spectral signatures (Fig. 7a-c). However, the current achievable coding capacities are still restricted due to the fact that the peaks of the commonly used SERS reporters are mostly located in the fingerprint region $\left(500-2000 \mathrm{~cm}^{-1}\right),{ }^{40}$ which results in crowding and indistinguishable Raman peaks.

Recently, Gao and co-workers developed super-capacity information-carrying systems by tuning vibrational signal intensities in multiple bands of Raman-silent regions. ${ }^{41}$ They generated a library of alkyne compounds where the Raman shifts located from 2000 to $2300 \mathrm{~cm}^{-1}$. The different Raman codes can be effectively generated through mixing the compounds with different amount ratios, thereby generating the distinct relative Raman intensities (Fig. 7d and e). This novel approach could experimentally yield the largest capacity of distinct optical barcodes, indicating that the Raman coding method provides a new strategy for super-capacity data storage and opens new scenarios for the development of high-throughput screening and data storage. 
a
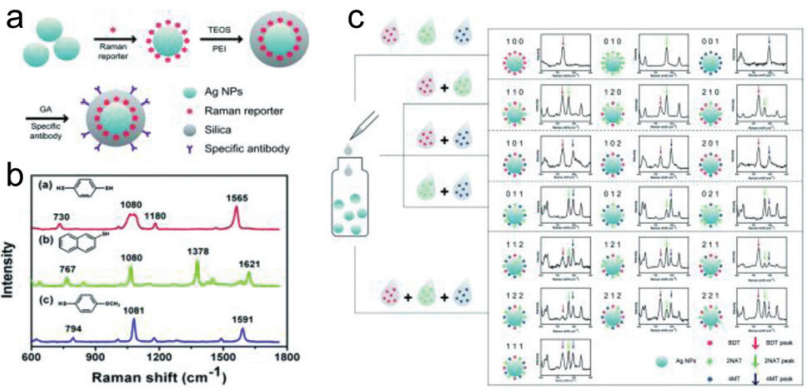

d

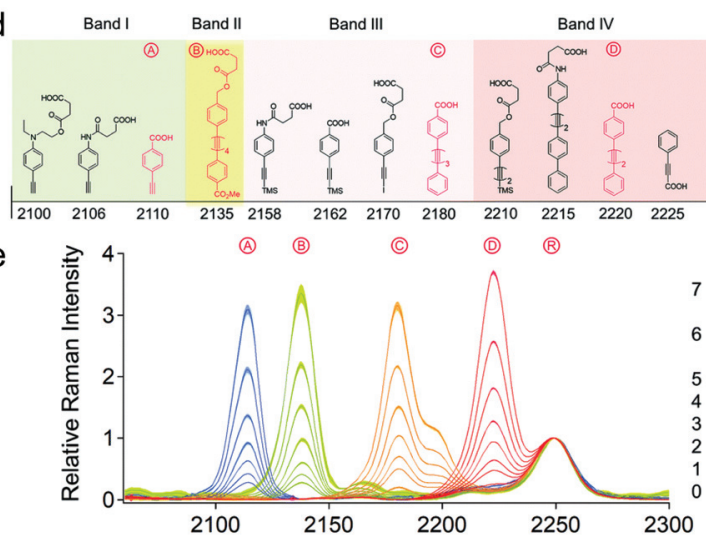

Fig. 7 (a) Schematic illustration of the preparation of the encoded SERS nanoprobes. Silver nanospheres were used as the SERS-active substrates, on which Raman reporters were adsorbed as the coding agents. Then, a layer of silica shell was coated for both protecting Raman reporters from the surroundings and providing a platform for further surface modifications. (b) SERS spectra of three SERS nanoprobes and the chemical structures of encoded Raman reporters: BDT, 2-NAT and 4-MT. (c) Codes, structures, and measured spectra of the synthesized 19 SERS encoders. The spectra are only shown from wavenumber 600 to 900 for clarity. (d) Molecular structures and Raman spectral properties of alkyne candidates for super-capacity coding systems. They are categorized into 4 spectral bands: bands I, II, III, and IV. Experimentally, these compounds labeled with $\left(A, B,\left(\subset, D_{1}\right.\right.$, and ${ }^{\circledR}$ were selected to covalently attach to aminolated resin beads. (e) Eight Raman codes generated through mixing the compounds with different amount ratios. They correspond to $\AA_{n}(n=0-7)$ at $2110 \mathrm{~cm}^{-1}, \Theta_{n}(n=0-7)$ at $2135 \mathrm{~cm}^{-1}, \Theta_{n}$ $(n=0-7)$ at $2180 \mathrm{~cm}^{-1}$, and $\triangle_{n}(n=0-7)$ at $2220 \mathrm{~cm}^{-1}$, respectively. (ac) Reproduced with permission from ref. 39. Copyright 2014, Royal Society of Chemistry. ( $d$ and e) Reproduced with permission from ref. 41. Copyright 2020, Royal Society of Chemistry.

Spectroscopic barcodes offer a facile, highly effective approach to information storage. Fluorescence is a principal element for expressing the identity of targets, which provides a wide range of fluorescent colors. The fluorescence would be modulated when organic luminophores are incorporated into a resonator, leading to recognizable peaks imposed on broad fluorescence emissions. The as-generated sharp peaks, similar to Raman scattering, contain fingerprint information of the individual resonator, providing a good candidate to improve the reliability of photonic barcodes.

\subsection{Integration of spectroscopic and graphical encoding}

Spectroscopic encoding methods will continue to be used at smaller scales because of the simplicity of code generation and detection. Besides, such a technique fits well with existing well-proven fluorescence detection devices, which no doubt would boost the applications, especially in the field of chemistry and life sciences. In this section, the integration of the spectroscopic and graphical encoding in organic materials is presented, where the advantages of identifiable microscale patterns and distinguishable spectral characteristics in organic materials would simultaneously be utilized, thereby greatly enlarging the encoding capacity and improving the security level.

The aim was fulfilled by Zhao and co-workers. ${ }^{42}$ They developed a strategy to design microscale barcodes with high coding capacity through integrating the spectroscopic and graphical encoding in 1D lanthanide metal-organic framework (Ln-MOF) multiblock heterostructures, which were fabricated via a facile stepwise solvothermal synthesis (Fig. 8a). The segregated Ln-MOF heterostructures with recognizable features and
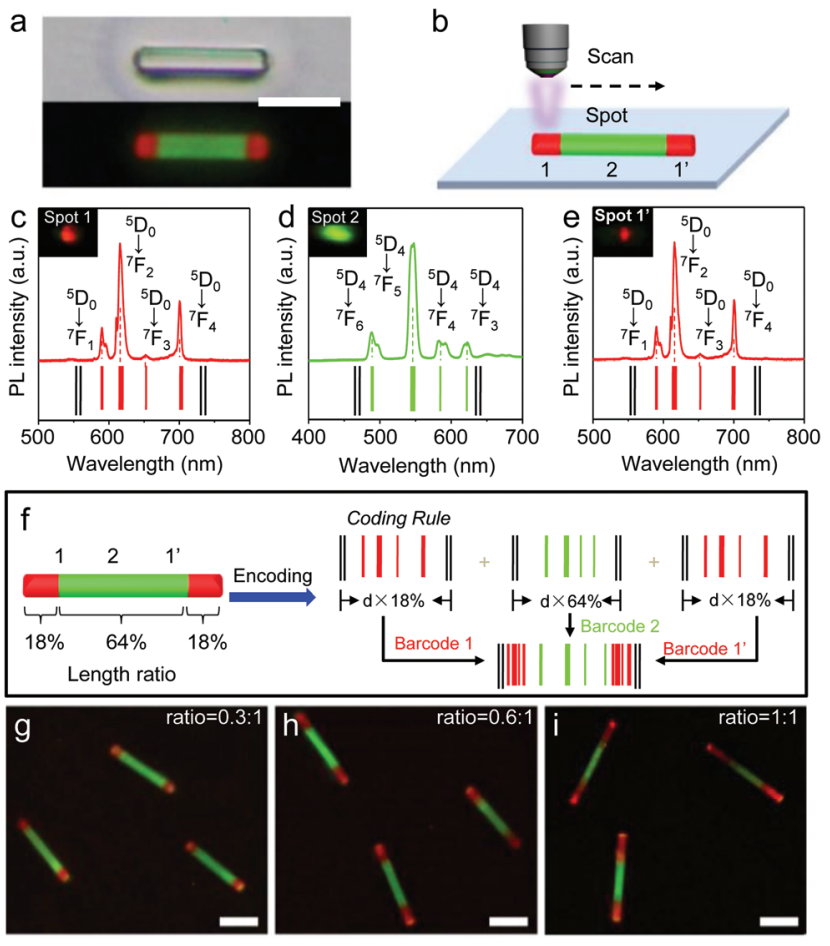

Fig. 8 (a) Bright-field and PL microscopy images of a segregated LnMOF heterostructure. Scale bar is $10 \mu \mathrm{m}$. (b) Schematic illustration of a heterostructure excited with a focused laser beam. Each part (marked as $\left.1,2,1^{\prime}\right)$ of the heterostructure was selectively excited with a CW laser beam $(375 \mathrm{~nm}$ ). (c-e) Corresponding PL spectra collected from three different areas and the generated barcodes (marked as 1, 2, 1' shown in b). (f) Schematic illustration of the encoding strategy based on the segregated Ln-MOF heterostructure. According to the different block lengths in a heterostructure, the as-designed barcodes were compressed with the corresponding length ratio, which can be defined as the sub-barcodes. The barcode was subsequently generated by combining the three parts of the sub-barcodes. (g-i) PL images of the segmented structure with various block lengths. The block length can be precisely controlled by adjusting the epitaxy precursor-to-seed ratio in the reaction system. Scale bars are $10 \mu \mathrm{m}$. Reproduced with permission from ref. 42. Copyright 2019, Wiley-VCH. 
distinguishable spectra constitute the fingerprint of a specific microstructure. Therefore, one can distinguish each microstructure based on its intrinsic PL spectrum, just like the recognition of the commodity by a specific barcode. These wellresolved PL spectra endow the multicolor Ln-MOF heterostructures with distinguishable features for developing spectroscopic encoding. Therefore, the PL spectrum collected from each segment can be expediently converted into a specific photonic barcode (Fig. 8b-e), which is beneficial for digital identification of the heterostructure.

Apart from the spectroscopic encoding, the different color lengths in each segment endow the heterostructure with a length-encoded character, providing a good platform to develop novel graphical encoding. As shown in Fig. 8f, based on different block lengths in the heterostructure, the asdesigned barcodes were compressed according to their corresponding length ratio. Then the built-up barcode was further generated by combining the different compressed sub-barcodes. By integrating the spectroscopic and graphical encoding, a specific barcode can be deduced from an individual LnMOF heterostructure, which allows for a one-to-one match between each multicolor barcode and the Ln-MOF heterostructure. Moreover, the optimized assembly method enables the production of countless unique barcodes through controllably fabricating diverse Ln-MOF heterostructures (Fig. 8g-i).

The integration of the spectroscopic and graphical encoding greatly enlarges the encoding capacity and improves the security level, providing enlightenment for the rational design of novel microstructures for data recording and information security.

\section{Covert photonic barcodes for advanced anti-counterfeiting}

Although micro/nanoscale barcodes have been widely used as object tags, the visible feature makes these barcodes easy to be altered or duplicated, facing increasing challenges as security labels in anti-counterfeiting applications. Covert photonic barcodes in which useful information is usually hidden until the predesigned reading method is conducted, would be a good addition to the aforementioned visible counterparts for anticounterfeiting and information security. ${ }^{43}$ This section discusses coding strategies of the covert photonic barcodes based on the abundant excited state processes with the near-infrared (NIR) luminescence mode and smart responsiveness to external stimuli, which are of great significance for optical data recording, anti-counterfeiting, and security labels.

\subsection{Covert photonic barcodes with near-infrared luminescence}

Conventional photonic barcodes are unfavorable for practical applications in confidential information protection, because the data or information recorded in these barcodes are usually visible under ambient or ordinary excitation of UV light. The near-infrared (NIR) luminescence is invisible to the naked eye, which provides an extra security feature and plays crucial roles in data communication and information security. ${ }^{44}$

Stéphane Petoud and co-workers proposed a new conceptual approach for creating luminescent barcoded systems based on NIR-emitting lanthanide MOFs. ${ }^{45}$ In this work, $\mathrm{Yb}^{3+}$ and $\mathrm{Er}^{3+}$ were chosen as the metal ions considering their distinguishable emission profiles in the NIR. The organic ligand of $\mathrm{H}_{2}$-PVDC can effectively sensitize lanthanide cations resulting from the efficient ligand-to-metal energy transfer (Fig. 9a). Excitation at a single wavelength produces the characteristic $\mathrm{Yb}^{3+}$ emission band centered at $980 \mathrm{~nm}$ and the $\mathrm{Er}^{3+}$ band centered at $1530 \mathrm{~nm}$ simultaneously. With an increase in the amount of $\mathrm{Er}^{3+}$ and a decrease in the amount of $\mathrm{Yb}^{3+}$, their respective emission intensities increase and decrease accordingly.

The different lanthanide metal ions exhibit similar coordination chemistry and atomic radii; thus different Ln-MOFs
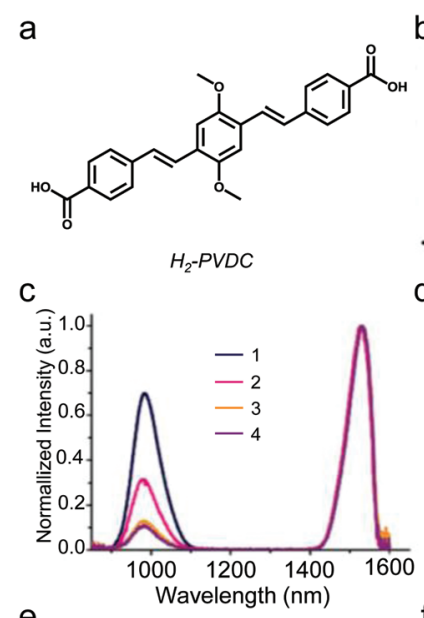

e

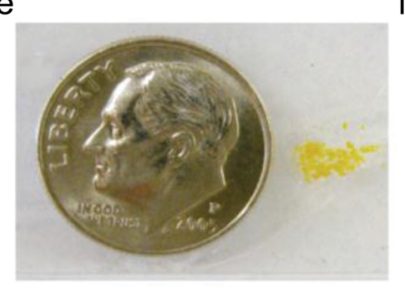

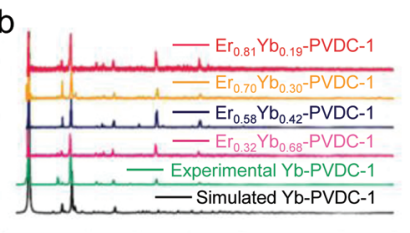

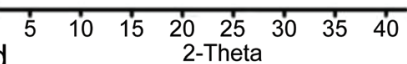

d

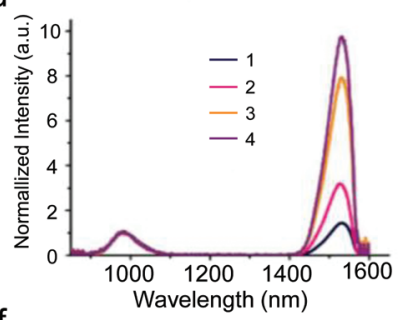

f

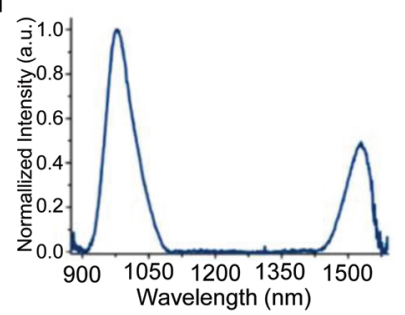

Fig. 9 (a) Molecular structure of the $\mathrm{H}_{2}$-PVDC, which was used to create polymetallic materials containing several different near-IR-emitting lanthanide cations. (b) Powder XRD patterns for Yb-PVDC-1 and other four luminescent metal-organic frameworks with varying lanthanide ion stoichiometries. (c and d) $\mathrm{Yb}^{3+}(980 \mathrm{~nm})$ and $\mathrm{Er}^{3+}(1530 \mathrm{~nm})$ emission spectra recorded upon $490 \mathrm{~nm}$ excitation: (c) normalized to the $\mathrm{Er}^{3+}$ signal; (d) normalized to the $\mathrm{Yb}^{3+}$ signal. With an increase in the amount of $\mathrm{Er}^{3+}$ and a decrease in the amount of $\mathrm{Yb}^{3+}$, their respective emission intensities increase and decrease accordingly. (e) Anti-counterfeiting demonstration of the NIR barcoded materials. Sample of $\mathrm{Er}_{0.70} \mathrm{Yb}_{0.30}-\mathrm{PVDC}-1$ needle crystals was dried and glued to a microscope slide (the dime is included for size perspective). (f) Corresponding $\mathrm{PL}$ spectrum of the sample shown in (e). Upon excitation at $490 \mathrm{~nm}$, the $\mathrm{Yb}^{3+}(980 \mathrm{~nm})$ and $\mathrm{Er}^{3+}(1530 \mathrm{~nm})$ barcode was easily detected in the NIR range. Reproduced with permission from ref. 45. Copyright 2009, American Chemical Society. 
show isomorphous structures (Fig. 9b). ${ }^{46}$ The heterobimetallic MOFs can quantitatively control the resulting luminescence intensities of the individual signals of the two NIRemitting lanthanide cations by controlling the lanthanide composition (Fig. 9c and d), which greatly enlarges the encoding capacity of the covert photonic barcodes. In addition, the luminescence bands in the NIR range can not only be perceived with the naked eye, but also be monitored with infrared sensors, which provides a good platform to develop high-level security labels towards anti-counterfeiting applications (Fig. 9e and $\mathrm{f}$ ).

\subsection{Covert photonic barcodes with smart responsiveness to external stimuli}

The static luminescence signals outputted by the current micro/nanoscale barcodes would hinder their practical applications for confidential information protection, because the data or information are usually visible under the ordinary excitation of UV or NIR light. Thus, barcodes with smart responses would play an indispensible role in preventing tampering or counterfeiting due to their additional advantages.

The abundant excited state processes in organic materials exhibit smart responsiveness to external stimuli and possess extra security features, affording an opportunity to develop covert photonic barcodes. We recently developed a strategy to construct the covert photonic barcodes based on the smart responsive organic WGM microcavities. ${ }^{32}$ The excellent compatibility of organic materials endows the WGM microcavities with responsiveness to external stimuli by doping smart responsive gain materials, which is highly desired in the construction of covert photonic barcodes. Coumarin 6 (C6) was adopted as an intelligent material due to its peculiar multiple excited states. It is easy for the C6 molecule to be associated with a proton in its thiazole ring nitrogen due to the basicity of this site (Fig. 10a). The protonation-induced changes in electronic properties can enhance the intramolecular charge transfer and induce a red-shift in the optical spectra in protonated C6 (Fig. 10b).

To generate the proton in an environment-friendly way, a photoacid generator (PAG), ${ }^{47}$ which can release a proton under illumination of light with a certain wavelength, was utilized to give rise to the protonated C6 molecules (Fig. 10c). Therefore, the microdisks manifest smart responsiveness to $254 \mathrm{~nm}$ light by taking $\mathrm{C} 6$ and PAG as the dopants, providing a good platform to create covert photonic barcodes that the real information would not appear until predesigned light stimuli are adopted (Fig. 10d). Interestingly, the composite microdisks changed their fluorescence color from cyan to green under the irradiation from a hand-held UV lamp (Fig. 10e and f). The irradiated microdisks with protonated C6 molecules emit another unique PL with a series of sharp peaks (Fig. 10g). Based on the proposed encoding rule, another photonic barcode associated with the UV-irradiated microdisk has been obtained, and it is different from the original one. This new barcode can be covert and form a "security tag", which can provide additional security protection. In comparison with

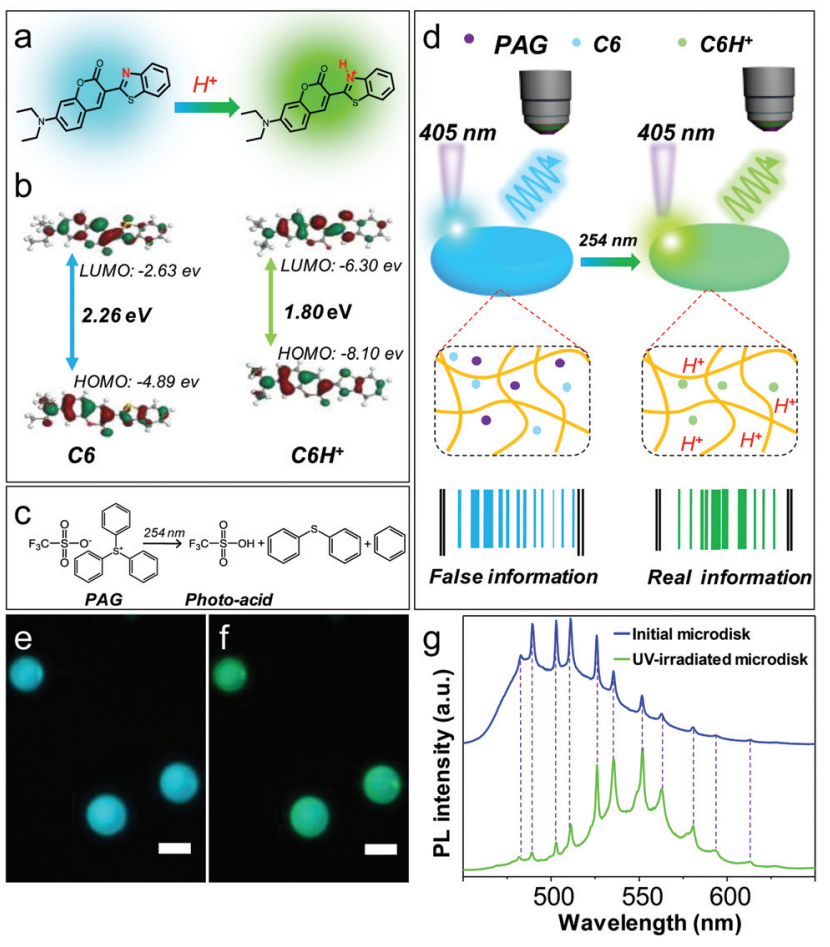

Fig. 10 (a) Molecular structures of $\mathrm{C} 6$ in neutral and protonated states, respectively. As a typical ICT dye, C6 can be regarded as an intelligent material due to its peculiar multiple excited states and sensitive response to environmental variations. (b) The corresponding pictorial presentation of the HOMO and LUMO of neutral and protonated C6 molecules. The protonation reduces the HOMO-LUMO gap from 2.26 to $1.80 \mathrm{eV}$, leading to a red-shift in optical spectra. (c) The reaction pathway of the PAG, which can release a proton under illumination of light with a certain wavelength. (d) Schematic diagram of a smart microdisk codoped with C6 and PAG for the covert photonic barcode. Under the irradiation from a UV lamp (254 nm), the composite microdisks simultaneously embodying $\mathrm{C} 6$ and PAG changed their fluorescence color from cyan to green, confirming that the photogenerated protons from PAG surely promote $\mathrm{C} 6$ to its protonated state. Only in this way, the real information can be read out. (e and f) PL images of microdisks with (e) neutral $\mathrm{C} 6$ and (f) protonated $\mathrm{C} 6$ molecules excited with the UV band $(330-380 \mathrm{~nm})$ of a mercury lamp. Scale bars are $10 \mu \mathrm{m}$. (g) PL spectra collected from the initial microdisk (blue) and the UV-irradiated microdisk (green) excited with a $405 \mathrm{CW}$ laser. The resonant wavelengths of the microdisk remain unchanged, presenting the ability to form a "security tag". Reproduced with permission from ref. 32. Copyright 2017, Wiley-VCH.

covert barcodes based on near-infrared (NIR) luminescence, the barcodes based on smart responses to external stimuli show a much higher security.

Barcode technology was first innovated and adopted in industry. Due to the robust practicability, applications of barcodes have spread to many areas including clinical medicine, food security, chemical detection, environmental monitoring, and retail-trade. One of the important applications of barcodes is multiplexed detection, which integrates multiple variables to overcome the clinical sensitivity and/or specificity limitations found with a single marker. ${ }^{2}$ The multiplexed detection based on the fluorescence color and intensity is the most 
Table 1 Comparison of different encoding methods

\begin{tabular}{|c|c|c|c|c|}
\hline & Encoding elements & Encoding capacity & Identification accuracy & Security level \\
\hline \multirow[t]{2}{*}{ Graphical coding } & Pattern & Unlimited number & + & + \\
\hline & Color and intensity & + & ++ & + \\
\hline \multirow[t]{2}{*}{ Spectroscopic encoding } & Microcavity modulation & ++ & +++ & ++ \\
\hline & Raman scattering & +++ & ++++ & ++ \\
\hline Integration of spectroscopic and graphical encoding & Color, intensity, pattern & +++ & +++ & +++ \\
\hline \multirow[t]{2}{*}{ Convert photonic barcodes } & Near-infrared & ++ & ++ & ++++ \\
\hline & Microcavity modulation & ++++ & +++ & +++++ \\
\hline
\end{tabular}

popular method due to its ease of visualization. ${ }^{48}$ Organic materials with the advantages of intense photoluminescence, chemical versatility, and excellent compatibility make them ideal candidates for multiplexed detection. Fluorescent dyes are generally applied as barcode materials to report the targets. Mixing different fluorescent dyes in various ratios is a useful strategy. ${ }^{49}$ Fluorescent micro/nanostructures with controlled size, morphology, constituents, and surface modification are available for different purposes in multiplex biosensing. Thus organic materials present great potential in designing barcodes for multiplexed detection.

\section{Outlook and perspectives}

The recent progress in micro/nanoscale photonic barcodes based on organic materials has been systematically summarized from the aspects of the diverse optical encoding techniques including identifiable organic microstructures, distinguishable spectral characteristics and controlled excitedstate processes of organics toward the construction of covert photonic barcodes (Table 1). However, this field still faces many challenges, such as further miniaturization and improvement of the reliability of organic barcodes. First, the size of organic photonic barcodes is larger than their output wavelength caused by the diffraction limit, hindering further miniaturization toward the subwavelength scale. The strong coupling between plasmon/exciton and optical modes, which would greatly enhance the light confinement may help realize ultrasmall photonic barcodes. Second, although organic materials with excellent flexibility and processability have been utilized to construct various barcodes, they often suffer from low thermal stability resulting from their weak intermolecular interactions, which may set an obstacle in their practical applications under certain working conditions. This problem could be solved by developing novel organic hybrid materials such as MOFs which possess the excellent processability of organic materials and high stability of inorganic materials.

Research studies on organic micro/nanoscale photonic barcodes, which are tightly related to materials design, fabrication and excited-state processes, is still in a preliminary stage. We hope that the rapid development in the past decade will help see it blossom in the next stage with collective efforts of researchers from different academic communities of chemistry, materials science, and related fields.

\section{Conflicts of interest}

There are no conflicts to declare.

\section{Acknowledgements}

This work was supported financially by the Ministry of Science and Technology of China (Grant No. 2018YFA0704802) and the National Natural Science Foundation of China (Grant No. 21922307, 21773265, and 21905145).

\section{Notes and references}

1 S. Shikha, T. Salafi, J. Cheng and Y. Zhang, Versatile Design and Synthesis of Nano-Barcodes, Chem. Soc. Rev., 2017, 46, 7054-7093.

2 Y. Leng, K. Sun, X. Chen and W. Li, Suspension Arrays Based on Nanoparticle-Encoded Microspheres for HighThroughput Multiplexed Detection, Chem. Soc. Rev., 2015, 44, 5552-5595.

3 H. Zhang, D. Hua, C. Huang, S. K. Samal, R. Xiong, F. Sauvage, K. Braeckmans, K. Remaut and S. C. De Smedt, Materials and Technologies to Combat Counterfeiting of Pharmaceuticals: Current and Future Problem Tackling, Adv. Mater., 2020, 32, 1905486.

4 (a) K. B. Cederquist, S. L. Dean and C. D. Keating, Encoded Anisotropic Particles for Multiplexed Bioanalysis, WIREs Nanomed. Nanobiotechnol., 2010, 2, 578-600; (b) W. Ren, G. Lin, C. Clarke, J. Zhou and D. Jin, Optical Nanomaterials and Enabling Technologies for High-Security-Level Anticounterfeiting, Adv. Mater., 2020, 32, 1901430; (c) F. Bian, L. Sun, L. Cai, Y. Wang, Y. Wang and Y. Zhao, Colloidal Crystals from Microfluidics, Small, 2020, 16, 1903931; (d) Y. Zhao, Y. Cheng, L. Shang, J. Wang, Z. Xie and Z. Gu, Microfluidic Synthesis of Barcode Particles for Multiplex Assays, Small, 2015, 11, 151-174; (e) Y. Zhao, L. Shang, Y. Cheng and Z. Gu, Spherical Colloidal Photonic Crystals, Acc. Chem. Res., 2014, 47, 3632-3642; (f) W. Hong, Z. Yuan and X. Chen, Structural Color Materials for Optical Anticounterfeiting, Small, 2020, 16, 1907626.

5 (a) Y. Wu, Y. Zhong, B. Chu, B. Sun, B. Song, S. Wu, Y. Su and Y. He, Plant-derived Fluorescent Silicon Nanoparticles Featuring Excitation Wavelength-Dependent Fluorescence Spectra for Anti-Counterfeiting Applications, Chem. 
Commun., 2016, 52, 7047-7050; (b) B. Song, H. Wang, Y. Zhong, B. Chu, Y. Su and Y. He, Fluorescent and Magnetic Anti-Counterfeiting Realized by Biocompatible Multifunctional Silicon Nanoshuttle-Based Security Ink, Nanoscale, 2018, 10, 1617-1621; (c) W. Yao, Q. Tian and W. Wu, Tunable Emissions of Upconversion Fluorescence for Security Applications, Adv. Opt. Mater., 2019, 7, 1801171; (d) Q. Y. Lin, E. Palacios, W. Zhou, Z. Li, J. A. Mason, Z. Liu, H. Lin, P. C. Chen, V. P. Dravid, K. Aydin and C. A. Mirkin, DNA-Mediated Size-Selective Nanoparticle Assembly for Multiplexed Surface Encoding, Nano Lett., 2018, 18, 2645-2649.

6 (a) J. Clark and G. Lanzani, Organic Photonics for Communications, Nat. Photonics, 2010, 4, 438-446; (b) Y. Yan and Y. S. Zhao, Organic Nanophotonics: from Controllable Assembly of Functional Molecules to LowDimensional Materials with Desired Photonic Properties, Chem. Soc. Rev., 2014, 43, 4325-4340; (c) W. Zhang, J. Yao and Y. S. Zhao, Organic Micro/Nanoscale Lasers, Acc. Chem. Res., 2016, 49, 1691-1700; (d) C. Zhang, Y. Yan, Y. S. Zhao and J. Yao, From Molecular Design and Materials Construction to Organic Nanophotonic Devices, Acc. Chem. Res., 2014, 47, 3448-3458; (e) C. Zhang, C. L. Zou, Y. Zhao, C. H. Dong, C. Wei, H. Wang, Y. Liu, G. C. Guo, J. Yao and Y. S. Zhao, Organic Printed Photonics: from Microring Lasers to Integrated Circuits, Sci. Adv., 2015, 1, e1500257; (f) J. Gu, B. Yin, S. Fu, M. Feng, Z. Zhang, H. Dong, F. Gao and Y. S. Zhao, Surface Tension Driven Aggregation of Organic Nanowires via Lab in a Droplet, Nanoscale, 2018, 10, 11006-11012.

7 M. Schubert, A. Steude, P. Liehm, N. M. Kronenberg, M. Karl, E. C. Campbell, S. J. Powis and M. C. Gather, Lasing within Live Cells Containing Intracellular Optical Microresonators for Barcode-Type Cell Tagging and Tracking, Nano Lett., 2015, 15, 5647-5652.

8 (a) Y. Lv, Y. J. Li, J. Li, Y. Yan, J. Yao and Y. S. Zhao, AllColor Subwavelength Output of Organic Flexible Microlasers, J. Am. Chem. Soc., 2017, 139, 11329-11332; (b) Z. Z. Li, F. Liang, M. P. Zhuo, Y. L. Shi, X. D. Wang and L. S. Liao, White-Emissive Self-Assembled Organic Microcrystals, Small, 2017, 13, 1604110.

9 L. Xu, W. Yan, W. Ma, H. Kuang, X. Wu, L. Liu, Y. Zhao, L. Wang and C. Xu, SERS Encoded Silver Pyramids for Attomolar Detection of Multiplexed Disease Biomarkers, Adv. Mater., 2015, 27, 1706-1711.

10 D. S.-z. Zhang, Y. Jiang, H. Yang, Y. Zhu, S. Zhang, Y. Zhu, D. Wei, Y. Lin, P. Wang, Q. Fu, H. Xu and H. Gu, DualEncoded Microbeads through a Host-Guest Structure: Enormous, Flexible, and Accurate Barcodes for Multiplexed Assays, Adv. Funct. Mater., 2016, 26, 6146-6157.

11 (a) K. Wang, W. Zhang, Z. Gao, Y. Yan, X. Lin, H. Dong, C. Zhang, W. Zhang, J. Yao and Y. S. Zhao, Stimulated Emission-Controlled Photonic Transistor on a Single Organic Triblock Nanowire, J. Am. Chem. Soc., 2018, 140, 13147-13150; (b) B. Yin, J. Gu, M. Feng, G. C. Zhang, Z. Zhang, J. Zhong, C. Zhang, B. Wen and Y. S. Zhao,
Epitaxial Growth of Dual-Color-Emitting Organic Heterostructures via Binary Solvent Synergism Driven Sequential Crystallization, Nanoscale, 2019, 11, 7111-7116; (c) M. P. Zhuo, J. J. Wu, X. D. Wang, Y. C. Tao, Y. Yuan and L.-S. Liao, Hierarchical Self-Assembly of Organic Heterostructure Nanowires, Nat. Commun., 2019, 10, 3839.

12 W. Zhang, Y. Yan, J. Gu, J. Yao and Y. S. Zhao, LowThreshold Wavelength-Switchable Organic Nanowire Lasers Based on Excited-State Intramolecular Proton Transfer, Angew. Chem., Int. Ed., 2015, 54, 7125-7129.

13 (a) H. Dong, Y. Wei, W. Zhang, C. Wei, C. Zhang, J. Yao and Y. S. Zhao, Broadband Tunable Microlasers Based on Controlled Intramolecular Charge-Transfer Process in Organic Supramolecular Microcrystals, J. Am. Chem. Soc., 2016, 138, 1118-1121; (b) B. Xu, Z. Gao, Y. Wei, Y. Liu, X. Sun, W. Zhang, X. Wang, Z. Wang and X. Meng, Dynamically Wavelength-Tunable Random Lasers Based on Metal-Organic Framework Particles, Nanoscale, 2020, 12, 4833-4838.

14 C. Zhang, C.-L. Zou, Y. Yan, R. Hao, F.-W. Sun, Z.-F. Han, Y. S. Zhao and J. Yao, Two-Photon Pumped Lasing in Single-Crystal Organic Nanowire Exciton Polariton Resonators, J. Am. Chem. Soc., 2011, 133, 7276-7279.

15 (a) Z. Gao, W. Zhang, Y. Yan, J. Yi, H. Dong, K. Wang, J. Yao and Y. S. Zhao, Proton-Controlled Organic Microlaser Switch, ACS Nano, 2018, 12, 5734-5740; (b) Y. Wei, H. Dong, C. Wei, W. Zhang, Y. Yan and Y. S. Zhao, Wavelength-Tunable Microlasers Based on the Encapsulation of Organic Dye in Metal-Organic Frameworks, Adv. Mater., 2016, 28, 7424-7429.

16 (a) X. Li, Y. Xie, B. Song, H. L. Zhang, H. Chen, H. Cai, W. Liu and Y. Tang, A Stimuli-Responsive Smart Lanthanide Nanocomposite for Multidimensional Optical Recording and Encryption, Angew. Chem., Int. Ed., 2017, 56, 2689-2693; (b) X. Hou, C. Ke, C. J. Bruns, P. R. McGonigal, R. B. Pettman and J. F. Stoddart, Tunable Solid-State Fluorescent Materials for Supramolecular Encryption, Nat. Commun., 2015, 6, 6884.

17 J. Lee, P. W. Bisso, R. L. Srinivas, J. J. Kim, A. J. Swiston and P. S. Doyle, Universal Process-Inert Encoding Architecture for Polymer Microparticles, Nat. Mater., 2014, 13, 524-529.

18 S. R. Nicewarner-Peña, R. G. Freeman, B. D. Reiss, L. He, D. J. Peña, I. D. Walton, R. Cromer, C. D. Keating and M. J. Natan, Submicrometer Metallic Barcodes, Science, 2001, 294, 137-141.

19 H. Xia, J. Cheng, L. Zhu, K. Xie, Q. Zhang, D. Zhang and G. Zou, One-Dimensional Programmable Polymeric Microfiber Waveguide with Optically Reconfigurable Photonic Functions, ACS Appl. Mater. Interfaces, 2019, 11, 15969-15976.

20 C. Zhang, Y. Yan, Y.-Y. Jing, Q. Shi, Y. S. Zhao and J. Yao, One-Dimensional Organic Photonic Heterostructures: Rational Construction and Spatial Engineering of Excitonic Emission, Adv. Mater., 2012, 24, 1703-1708.

21 K. Wang, H. Zhang, S. Chen, G. Yang, J. Zhang, W. Tian, Z. Su and Y. Wang, Organic Polymorphs: One-Compound- 
Based Crystals with Molecular-Conformation- and PackingDependent Luminescent Properties, Adv. Mater., 2014, 26, 6168-6173.

22 C. Zhang, Y. Yan, J. Yao and Y. S. Zhao, Manipulation of Light Flows in Organic Color-Graded Microstructures towards Integrated Photonic Heterojunction Devices, Adv. Mater., 2013, 25, 2854-2859.

23 M.-J. Sun, Y. Liu, Y. Yan, R. Li, Q. Shi, Y. S. Zhao, Y.-W. Zhong and J. Yao, In Situ Visualization of Assembly and Photonic Signal Processing in a Triplet LightHarvesting Nanosystem, J. Am. Chem. Soc., 2018, 140, 42694278.

24 X. Ji, R.-T. Wu, L. Long, X.-S. Ke, C. Guo, Y.-J. Ghang, V. M. Lynch, F. Huang and J. L. Sessler, Encoding, Reading, and Transforming Information Using Multifluorescent Supramolecular Polymeric Hydrogels, Adv. Mater., 2018, 30, 1705480 .

25 (a) K. Braeckmans, S. C. De Smedt, C. Roelant, M. Leblans, R. Pauwels and J. Demeester, Encoding Microcarriers by Spatial Selective Photobleaching, Nat. Mater., 2003, 2, 169173; (b) F. Fayazpour, B. Lucas, N. Huyghebaert, K. Braeckmans, S. Derveaux, B. G. Stubbe, J.-P. Remon, J. Demeester, C. Vervaet and S. C. De Smedt, Digitally Encoded Drug Tablets to Combat Counterfeiting, Adv. Mater., 2007, 19, 3854-3858.

26 C. Huang, B. Lucas, C. Vervaet, K. Braeckmans, S. Van Calenbergh, I. Karalic, M. Vandewoestyne, D. Deforce, J. Demeester and S. C. De Smedt, Unbreakable Codes in Electrospun Fibers: Digitally Encoded Polymers to Stop Medicine Counterfeiting, Adv. Mater., 2010, 22, 2657-2662.

27 K. Braeckmans, S. C. De Smedt, M. Leblans, R. Pauwels and J. Demeester, Encoding Microcarriers: Present and Future Technologies, Nat. Rev. Drug Discovery, 2002, 1, 447456.

28 (a) H. H. Gorris, R. Ali, S. M. Saleh and O. S. Wolfbeis, Tuning the Dual Emission of Photon-Upconverting Nanoparticles for Ratiometric Multiplexed Encoding, Adv. Mater., 2011, 23, 1652-1655; (b) Y. Lu and B. Yan, Luminescent Lanthanide Barcodes Based on Postsynthetic Modified Nanoscale Metal-Organic Frameworks, J. Mater. Chem. C, 2014, 2, 7411-7416; (c) D. Kage, K. Hoffmann, M. Wittkamp, J. Ameskamp, W. Göhde and U. ReschGenger, Luminescence Lifetime Encoding in Time-Domain Flow Cytometry, Sci. Rep., 2018, 8, 16715.

29 S. Park, J. E. Kwon, S. H. Kim, J. Seo, K. Chung, S.-Y. Park, D.-J. Jang, B. M. Medina, J. Gierschner and S. Y. Park, A White-Light-Emitting Molecule: Frustrated Energy Transfer between Constituent Emitting Centers, J. Am. Chem. Soc., 2009, 131, 14043-14049.

30 X. Zhang, Y. Xiao, L. He and Y. Zhang, Through-Bond Energy Transfer Cassettes for Multicolor Encoding, J. Org. Chem., 2014, 79, 6315-6320.

$31 \mathrm{H}$. H. Gorris and O. S. Wolfbeis, Photon-Upconverting Nanoparticles for Optical Encoding and Multiplexing of Cells, Biomolecules, and Microspheres, Angew. Chem., Int. Ed., 2013, 52, 3584-3600.
32 Z. Gao, C. Wei, Y. Yan, W. Zhang, H. Dong, J. Zhao, J. Yi, C. Zhang, Y. J. Li and Y. S. Zhao, Covert Photonic Barcodes Based on Light Controlled Acidichromism in Organic Dye Doped Whispering-Gallery-Mode Microdisks, Adv. Mater., 2017, 29, 1701558.

33 K. J. Vahala, Optical Microcavities, Nature, 2003, 424, 839846.

34 F. Ramiro-Manzano, R. Fenollosa, E. Xifré-Pérez, M. Garín and F. Meseguer, Porous Silicon Microcavities Based Photonic Barcodes, Adv. Mater., 2011, 23, 3022-3025.

35 (a) C. Wei, S. Y. Liu, C. L. Zou, Y. Liu, J. Yao and Y. S. Zhao, Controlled Self-Assembly of Organic Composite Microdisks for Efficient Output Coupling of Whispering-Gallery-Mode Lasers, J. Am. Chem. Soc., 2015, 137, 62-65; (b) C. Wei, Y. Du, Y. Liu, X. Lin, C. Zhang, J. Yao and Y. S. Zhao, Organic Janus Microspheres: A General Approach to AllColor Dual-Wavelength Microlasers, J. Am. Chem. Soc., 2019, 141, 5116-5120; (c) C. Wei, M. Gao, F. Hu, J. Yao and Y. S. Zhao, Excimer Emission in Self-Assembled Organic Spherical Microstructures: An Effective Approach to Wavelength Switchable Microlasers, Adv. Opt. Mater., 2016, 4, 1009-1014.

36 V. D. Ta, R. Chen and H. D. Sun, Self-Assembled Flexible Microlasers, Adv. Mater., 2012, 24, OP60-OP64.

37 J. Vogelsang, R. Kasper, C. Steinhauer, B. Person, M. Heilemann, M. Sauer and P. Tinnefeld, A Reducing and Oxidizing System Minimizes Photobleaching and Blinking of Fluorescent Dyes, Angew. Chem., Int. Ed., 2008, 47, 54655469.

38 (a) L. Rodriguez-Lorenzo, L. Fabris and R. A. AlvarezPuebla, Multiplex Optical Sensing with Surface-Enhanced Raman Scattering: A Critical Review, Anal. Chim. Acta, 2012, 745, 10-23; (b) Y. Liu and P. Wu, Meditating Metal Coenhanced Fluorescence and SERS Around Gold Nanoaggregates in Nanosphere as Bifunctional Biosensor for Multiple DNA Targets, ACS Appl. Mater. Interfaces, 2013, 5, 5832-5844; (c) Y. Lai, S. Sun, T. He, S. Schlücker and Y. Wang, Raman-Encoded Microbeads for Spectral Multiplexing with SERS Detection, RSC Adv., 2015, 5, 13762-13767.

39 D. Zhu, Z. Wang, S. Zong, H. Chen, P. Chen and Y. Cui, Wavenumber-Intensity Joint SERS Encoding Using Silver Nanoparticles for Tumor Cell Targeting, RSC Adv., 2014, 4, 60936-60942.

40 Z. Wang, S. Zong, L. Wu, D. Zhu and Y. Cui, SERS-Activated Platforms for Immunoassay: Probes, Encoding Methods, and Applications, Chem. Rev., 2017, 117, 7910-7963.

41 Y. Tang, C. He, X. Zheng, X. Chen and T. Gao, SuperCapacity Information-Carrying Systems Encoded with Spontaneous Raman Scattering, Chem. Sci., 2020, 11, 30963103.

42 Y. Yao, Z. Gao, Y. Lv, X. Lin, Y. Liu, Y. Du, F. Hu and Y. S. Zhao, Heteroepitaxial Growth of Multiblock Ln-MOF Microrods for Photonic Barcodes, Angew. Chem., Int. Ed., 2019, 58, 13803-13807. 
43 B. Duong, H. Liu, L. Ma and M. Su, Covert Thermal Barcodes Based on Phase Change Nanoparticles, Sci. Rep., 2014, 4, 5170.

44 Y.-H. Chen, S.-Y. Kuo, W.-K. Tsai, C.-S. Ke, C.-H. Liao, C.-P. Chen, Y.-T. Wang, H.-W. Chen and Y.-H. Chan, Dual Colorimetric and Fluorescent Imaging of Latent Fingerprints on Both Porous and Nonporous Surfaces with Near-Infrared Fluorescent Semiconducting Polymer Dots, Anal. Chem., 2016, 88, 11616-11623.

45 K. A. White, D. A. Chengelis, K. A. Gogick, J. Stehman, N. L. Rosi and S. Petoud, Near-Infrared Luminescent Lanthanide MOF Barcodes, J. Am. Chem. Soc., 2009, 131, 18069-18071.

46 (a) B. Yan, Lanthanide-Functionalized Metal-Organic Framework Hybrid Systems To Create Multiple Luminescent Centers for Chemical Sensing, Acc. Chem. Res., 2017, 50, 2789-2798; (b) X. Yang, X. Lin, Y. Zhao,
Y. S. Zhao and D. Yan, Lanthanide Metal-Organic Framework Microrods: Colored Optical Waveguides and Chiral Polarized Emission, Angew. Chem., Int. Ed., 2017, 56, 7853-7857.

47 D. R. Whang, Y. You, W. S. Chae, J. Heo, S. Kim and S. Y. Park, Solid-state Phosphorescence-to-Fluorescence Switching in a Cyclometalated Ir(III) Complex Containing an Acid-Labile Chromophoric Ancillary Ligand: Implication for Multimodal Security Printing, Langmuir, 2012, 28, 15433-15437.

48 J. M. Levsky, S. M. Shenoy, R. C. Pezo and R. H. Singer, Single-Cell Gene Expression Profiling, Science, 2002, 297, 836-840.

49 Y. Li, Y. T. H. Cu and D. Luo, Multiplexed Detection of Pathogen DNA with DNA-Based Fluorescence Nanobarcodes, Nat. Biotechnol., 2005, 23, 885-889. 